\title{
Corrections to: Achieving Glycaemic Control with Concentrated Insulin in Patients with Type 2 Diabetes
}

\author{
Sudesna Chatterjee ${ }^{1,2}\left(\mathbb{D} \cdot\right.$ Kamlesh Khunti $^{3} \cdot$ Melanie J. Davies $^{3}$
}

Published online: 13 March 2019

C) Springer Nature Switzerland AG 2019

\section{Corrections to: Drugs (2019) 79:173-186 https://doi.org/10.1007/s40265-018-1048-6}

In several sections of this review article, insulin degludec U100 has been incorrectly referred to as a highly concentrated basal insulin. Although data for both the U100 and U200 formulations of insulin degludec are presented, only insulin degludec U200 and insulin glargine U300 should be referred to as highly concentrated basal insulins.

In Fig. 2 and in section 5.4, the information regarding insulin degludec initiation in patients already on insulin therapy was sourced from the US prescribing information (https://www.accessdata.fda.gov/drugsatfda_docs/label /2018/203314s010lbl.pdf), which states that adults with type 1 or type 2 diabetes who are already on insulin therapy start TRESIBA $®$ at the same unit dose as the total daily long or intermediate-acting insulin unit dose, while paediatric patients 1 year of age or older should start at $80 \%$ of the total daily long or intermediate-acting insulin unit dose to minimize the risk of hypoglycaemia. This differs from the EU product information (https://www.ema.europa.eu/en/ documents/product-information/tresiba-epar-product-infor mation_en.pdf), which states that a $20 \%$ dose reduction should be considered when transferring patients with type 2 diabetes from twice-daily basal insulin or insulin glargine U300, and for patients with type 1 diabetes based on their previous basal insulin dose or the basal component of a continuous subcutaneous insulin infusion regimen.

The original article can be found online at https://doi.org/10.1007/ s40265-018-1048-6.

Sudesna Chatterjee

Sudesna.chatterjee@abbott.com

1 University Hospitals of Leicester NHS Trust, Senior Clinical Researcher, University of Leicester, Leicester, UK

2 Present Address: Abbott Diabetes Care, Abbott Laboratories, Maidenhead, UK

3 Diabetes Research Centre, University of Leicester, Leicester, UK 